\title{
Fibonacci Polynomials and Determinant Identities
}

\author{
Omprakash Sikhwal ${ }^{1, *}$, Yashwant Vyas ${ }^{2}$ \\ ${ }^{1}$ Department of Mathematics, Mandsaur Institute of Technology, Mandsaur (M. P.), India \\ ${ }^{2}$ Department of Mathematics, Shri Harak Chand Chordia College, Bhanpura (M. P.), India \\ *Corresponding author: opbhsikhwal@rediffmail.com
}

Received September 03, 2014; Revised October 06, 2014; Accepted October 14, 2014

\begin{abstract}
The Fibonacci polynomials and Lucas polynomials are famous for possessing wonderful and amazing properties and identities. In this paper, some determinant identities of Fibonacci polynomials are describe. Entries of determinants are satisfying the recurrence relations of Fibonacci polynomials and Lucas polynomials.
\end{abstract}

\section{Keywords: Fibonacci number, Fibonacci polynomial, Lucas polynomial, determinant}

Cite This Article: Omprakash Sikhwal, and Yashwant Vyas, "Fibonacci Polynomials and Determinant Identities." Turkish Journal of Analysis and Number Theory, vol. 2, no. 6 (2014): 189-192. doi: 10.12691/tjant-2$5-6$.

\section{Introduction}

Fibonacci numbers are a popular topic for mathematical enrichment and popularization. They are famous for a host of interesting and surprising properties and show up in text books, magazine articles, and web sites. Various sequences of polynomials by the names of Fibonacci and Lucas polynomials occur in the literature over a century. The Fibonacci polynomials and Lucas polynomials are closely related and widely investigated. Fibonacci polynomials appear in different frameworks. Fibonacci polynomials are special cases of Chebyshev polynomials and have been studied on a more advanced level by many mathematicians.

S. L. Basin [15] show that Q matrix generates a set of Fibonacci Polynomials satisfying the recurrence relation

$$
\mathrm{f}_{\mathrm{n}+1}(x)=\mathrm{xf}_{\mathrm{n}}(x)+\mathrm{f}_{\mathrm{n}-1,},(\mathrm{x}), \mathrm{n} \geq 2
$$

with

$$
\mathrm{f}_{0}(x)=0, \mathrm{f}_{1}(x)=1 .
$$

The Lucas Polynomials [1] are defined by the recurrence formula

$$
1_{\mathrm{n}+1}(x)=\mathrm{xl}_{\mathrm{n}}(x)+1_{\mathrm{n}-1},(\mathrm{x}), \mathrm{n} \geq 2
$$

with

$$
\mathrm{l}_{0}(x)=2, \mathrm{l}_{1}(x)=\mathrm{x} .
$$

Generating function of Fibonacci polynomials is

$$
\sum_{n=0}^{\infty} f_{n}(x) t^{n}=t\left(1-x t-t^{2}\right)^{-1} .
$$

Generating function of Lucas polynomials is

$$
\sum_{n=0}^{\infty} l_{n}(x) t^{n}=(2-x t)\left(1-x t-t^{2}\right)^{-1} .
$$

Explicit sum formula for (1.1) is given by

$$
f_{n}(x)=\sum_{k=0}^{\left[\frac{n-1}{2}\right]}\left(\begin{array}{c}
n-k-1 \\
k
\end{array}\right) x^{n-1-2 k},
$$

where $\left(\begin{array}{l}n \\ m\end{array}\right)$ a binomial coefficient and $[\mathrm{x}]$ is define as the greatest integer less than or equal to $\mathrm{x}$.

Explicit sum formula for (1.2) is given by

$$
l_{n}(x)=\sum_{k=0}^{[n / 2]} \frac{n}{n-k}\left(\begin{array}{c}
n-k \\
k
\end{array}\right) x^{n-2 k},
$$

where $\left(\begin{array}{l}n \\ m\end{array}\right)$ a binomial coefficient and $[\mathrm{x}]$ is defined as the greatest integer less than or equal to $\mathrm{x}$.

Determinants have played a significant part in various areas in mathematics. For instance, they are quite useful in the analysis and solution of system of linear equations. There are different perspectives on the study of determinants. One may notice several practical and effective instruments for calculating determinants in the nice survey articles [7] and [8].

Much attention has been paid to the evaluation of determinants of matrices, especially when their entries are given recursively [8].

There is a long tradition of using matrices and determinants to study Fibonacci numbers. Bicknell Johnson and Spears [11] use elementary matrix operations and determinants to generate classes of identities for generalized Fibonacci numbers. Cahill and Narayan [12] show how Fibonacci and Lucas numbers arise as determinants of some tridiagonal matrices. A. Benjamin, $\mathrm{T}$. Cameron and J. Quinn [2], provides combinatorial interpretations for Fibonacci identities using determinants. T. Koshy [16] explained two chapters on the use of matrices and determinants in Fibonacci numbers. O. Sikhwal [13] explained determinants identities of Fibonacci sequences and its generalizations. 
The Fibonacci and Lucas polynomials possess many fascinating properties which have been studied in [1] to [9] and [11] to [15]. In this paper, some determinant identities of Fibonacci polynomials are describe.

\section{Determinan Identities}

We define a family of Fibonacci polynomial as $B=\left\{f_{n+p}(x), f_{n+q}(x), f_{n+q+r}(x), f_{n+s}(x), f_{n+s+r}(x)\right\}$, where $\mathrm{n}$ and $\mathrm{p}$ are non negative integers, $\mathrm{q}$ and $\mathrm{s}$ are positive integers with $0 \leq \mathrm{p}<\mathrm{q}, \mathrm{q}+1<\mathrm{s} r=1$.

Assume $f_{n+p}(x)=a, \quad f_{n+q}(x)=b$, then by $(1.1)$ $f_{n+q+r}(x)=a+b x, f_{n+q+r}(x)=x f_{n+q}+f_{n+p}$, $f_{n+s}(x)=x f_{n+q+r}+f_{n+q}, f_{n+s+r}(x)=x f_{n+s}(x)+f_{n+q+r}$.

Theorem 1: If $\mathrm{n}$ and $\mathrm{p}$ are non-negative integers, $\mathrm{q}$ is positive integer with $0 \leq \mathrm{p}<\mathrm{q}, \mathrm{r}=1$, Prove that

$$
\begin{aligned}
& \left|\begin{array}{ccc}
f_{n+p}(x) & f_{n+q}(x) & f_{n+q+r}(x) \\
f_{n+q+r}(x) & f_{n+p}(x) & f_{n+q}(x) \\
f_{n+q}(x) & f_{n+q+r}(x) & f_{n+p}(x)
\end{array}\right| \\
& =f_{n+p}^{3}(x)+f_{n+q}^{3}(x)+f_{n+q+r}^{3} \\
& (x)-3 f_{n+p}(x) f_{n+q}(x) f_{n+q+r}(x)
\end{aligned}
$$

Proof:

Let

$$
\Delta=\left|\begin{array}{ccc}
f_{n+p}(x) & f_{n+q}(x) & f_{n+q+r}(x) \\
f_{n+q+r}(x) & f_{n+p}(x) & f_{n+q}(x) \\
f_{n+q}(x) & f_{n+q+r}(x) & f_{n+p}(x)
\end{array}\right|
$$

Assume $f_{n+p}(x)=a, \quad f_{n+q}(x)=b$, then by $f_{n+q+r}(x)=a+b x$

Now

$$
\Delta=\left|\begin{array}{ccc}
a & b & a+b x \\
a+b x & a & b \\
b & a+b x & a
\end{array}\right|
$$

Applying $R_{1}+R_{2} \rightarrow R_{1}$,

$$
\Delta=\left|\begin{array}{ccc}
2 a+b x & a+b & a+b+b x \\
a+b x & a & b \\
b & a+b x & a
\end{array}\right|
$$

Applying $C_{1}-C_{2} \rightarrow C_{1}$,

$$
\Delta=\left|\begin{array}{ccc}
a+b x-b & a+b & a+b+b x \\
b x & a & b \\
b-a-b x & a+b x & a
\end{array}\right|
$$

Applying $R_{1}+R_{3} \rightarrow R_{1}$,

$$
\Delta=\left|\begin{array}{ccc}
0 & 2 a+b+b x & 2 a+b+b x \\
b x & a & b \\
b-a-b x & a+b x & a
\end{array}\right|
$$

Applying $C_{2}-C_{3} \rightarrow C_{2}$,

$$
\Delta=\left|\begin{array}{ccc}
0 & 0 & 2 a+b+b x \\
b x & a-b & b \\
b-a-b x & b x & a
\end{array}\right| .
$$

Expand along first row, we get

$$
\Delta=(a+b x)^{3}+a^{3}+b^{3}-3 a b(a+b x) .
$$

Put $f_{n+p}(x)=a, f_{n+q}(x)=b, f_{n+q+r}(x)=a+b x$, we get

$$
\begin{aligned}
& \left|\begin{array}{ccc}
f_{n+p}(x) & f_{n+q}(x) & f_{n+q+r}(x) \\
f_{n+q+r}(x) & f_{n+p}(x) & f_{n+q}(x) \\
f_{n+q}(x) & f_{n+q+r}(x) & f_{n+p}(x)
\end{array}\right| \\
& =f_{n+p}^{3}(x)+f_{n+q}^{3}(x)+f_{n+q+r}^{3}(x) \\
& -3 f_{n+p}(x) f_{n+q}(x) f_{n+q+r}(x) .
\end{aligned}
$$

Corollary 1.1: If we put $x=1$ in above result, for $0 \leq \mathrm{p}<\mathrm{q}$, $\mathrm{r}=1$, we get

$$
\begin{aligned}
& \left|\begin{array}{ccc}
F_{n+p} & F_{n+q} & F_{n+q+r} \\
F_{n+q+r} & F_{n+p} & F_{n+q} \\
F_{n+q} & F_{n+q+r} & F_{n+p}
\end{array}\right| \\
& =F_{n+p}^{3}+F_{n+q}^{3}+F_{n+q+r}^{3}-3 F_{n+p} F_{n+q} F_{n+q+r} \\
& =2\left(F_{n+p}^{3}+F_{n+q}^{3}\right) .
\end{aligned}
$$

It can be proved easily.

Theorem 2: If $\mathrm{n}$ and $\mathrm{p}$ are non-negative integers, $\mathrm{q}$ is positive integer with $0 \leq \mathrm{p}<\mathrm{q}, \mathrm{r}=1$, Prove that

$$
\begin{aligned}
& \left|\begin{array}{ccc}
f_{n+p}(x) & l_{n+p}(x) & 1 \\
f_{n+q}(x) & l_{n+q}(x) & 1 \\
f_{n+q+r}(x) & l_{n+q+r}(x) & 0
\end{array}\right| \\
& =(x+1)\left[f_{n+q}(x) l_{n+p}(x)-f_{n+p}(x) l_{n+q}(x)\right] \\
& =\left\{\begin{array}{c}
2(1+x), \text { if } n=0,2,4 \ldots \\
-2(1+x), \text { if } n=1,3,5, \ldots
\end{array}\right.
\end{aligned}
$$

Proof: Let $\Delta=\left|\begin{array}{ccc}f_{n+p}(x) & l_{n+p}(x) & 1 \\ f_{n+q}(x) & l_{n+q}(x) & 1 \\ f_{n+q+r}(x) & l_{n+q+r}(x) & 0\end{array}\right|$

Assume $f_{n+p}(x)=a, f_{n+q}(x)=b$, then by $(1.1)$ $f_{n+q+r}(x)=a+b x$ and $l_{n+p}(x)=c, l_{n+q}(x)=d$, then by (1.2), $l_{n+q+r}(x)=c+d x$.

Now 


$$
\Delta=\left|\begin{array}{ccc}
a & c & 1 \\
b & d & 1 \\
a+b x & c+d x & 0
\end{array}\right|
$$

Applying $R_{1}-R_{2} \rightarrow R_{1}$,

$$
\Delta=\left|\begin{array}{ccc}
a-b & c-d & 0 \\
b & d & 1 \\
a+b x & c+d x & 0
\end{array}\right|
$$

Interchanging $C_{1}$ and $C_{3}$,

$$
\Delta=(-1)\left|\begin{array}{ccc}
0 & c-d & a-b \\
1 & d & b \\
0 & c+d x & a+b x
\end{array}\right|
$$

Expand it, we get

$$
\Delta=(x+1)[b c-a d]
$$

Assume $f_{n+p}(x)=a, f_{n+q}(x)=b$, then by $(1.1)$ $f_{n+q+r}(x)=a+b x$ and then by $l_{n+p}(x)=c, l_{n+q}(x)=d l_{n+q+r}(x)=c+d x$, we get

$$
\begin{aligned}
& \left|\begin{array}{ccc}
f_{n+p}(x) & l_{n+p}(x) & 1 \\
f_{n+q}(x) & l_{n+q}(x) & 1 \\
f_{n+q+r}(x) & l_{n+q+r}(x) & 0
\end{array}\right| \\
& =(x+1)\left[f_{n+q}(x) l_{n+p}(x)-f_{n+p}(x) l_{n+q}(x)\right] \\
& =\left\{\begin{array}{c}
2(1+x), \text { if } n=0,2,4 \ldots \\
-2(1+x), \text { if } n=1,3,5, \ldots
\end{array}\right.
\end{aligned}
$$

Corollary 2.1: If we put $x=1$ in above result, we get

$$
\begin{aligned}
& \left|\begin{array}{ccc}
F_{n+p} & L_{n+p} & 1 \\
F_{n+q} & L_{n+p} & 1 \\
F_{n+q+r} & L_{n+q+r} & 0
\end{array}\right| \\
& =2\left[F_{n+q} L_{n+p}-F_{n+p} L_{n+q}\right]=\left\{\begin{array}{l}
4, n=0,2,4, \ldots \\
-4, n=1,3,5, \ldots
\end{array}\right.
\end{aligned}
$$

It can be proved easily.

Theorem 3: If $\mathrm{n}$ and $\mathrm{p}$ are non-negative integers, $\mathrm{q}$ and $\mathrm{s}$ are positive integers with $0 \leq \mathrm{p}<\mathrm{q}, \mathrm{q}+1<\mathrm{s} r=1$, Prove that

$$
\left|\begin{array}{ccc}
f_{n+p}(x) & f_{n+q}(x) & f_{n+q+r}(x) \\
f_{n+q}(x) & f_{n+q+r}(x) & f_{n+s}(x) \\
f_{n+q+r}(x) & f_{n+s}(x) & f_{n+s+r}(x)
\end{array}\right|=0
$$

Proof: Assume $f_{n+p}(x)=a, \quad f_{n+q}(x)=b$, then by (1.1) $f_{n+q+r}(x)=a+b x \quad$ and $\quad f_{n+q+r}(x)=x f_{n+q}+f_{n+p} \quad$, $f_{n+s}(x)=x f_{n+q+r}+f_{n+q}, f_{n+s+r}(x)=x f_{n+s}(x)+f_{n+q+r}$.

Let

$$
\Delta=\left|\begin{array}{ccc}
f_{n+p}(x) & f_{n+q}(x) & f_{n+q+r}(x) \\
f_{n+q}(x) & f_{n+q+r}(x) & f_{n+s}(x) \\
f_{n+q+r}(x) & f_{n+s}(x) & f_{n+s+r}(x)
\end{array}\right|
$$

Applying $C_{1}+x C_{2} \rightarrow C_{1}$,

$$
\Delta=\left|\begin{array}{ccc}
f_{n+q+r}(x) & f_{n+q}(x) & f_{n+q+r}(x) \\
f_{n+s}(x) & f_{n+q+r}(x) & f_{n+s}(x) \\
f_{n+s+r}(x) & f_{n+s}(x) & f_{n+s+r}(x)
\end{array}\right|
$$

Here two columns are identical, we get

$$
\Delta=\left|\begin{array}{ccc}
f_{n+p}(x) & f_{n+q}(x) & f_{n+q+r}(x) \\
f_{n+q}(x) & f_{n+q+r}(x) & f_{n+s}(x) \\
f_{n+q+r}(x) & f_{n+s}(x) & f_{n+s+r}(x)
\end{array}\right|=0
$$

Corollary 3.1: If we put $\mathrm{x}=1$ in above result, we get

$$
\left|\begin{array}{ccc}
F_{n+p} & F_{n+q} & F_{n+q+r} \\
F_{n+q} & F_{n+q+r} & F_{n+s} \\
F_{n+q+r} & F_{n+s} & F_{n+s+r}
\end{array}\right|=0
$$

It can be proved easily.

Theorem 4: If $\mathrm{n}$ and $\mathrm{p}$ are non-negative integers, $\mathrm{q}$ is positive integer with $0 \leq \mathrm{p}<\mathrm{q}, \mathrm{r}=1$, Prove that

$$
\begin{aligned}
& \left|\begin{array}{ccc}
\left(f_{n+p}(x)+f_{n+q}(x)\right)^{2} & f_{n+p}(x) f_{n+q+r}(x) & f_{n+q}(x) f_{n+q+r}(x) \\
f_{n+p}(x) f_{n+q+r}(x) & \left(f_{n+q}(x)+f_{n+q+r}(x)\right)^{2} & f_{n+p}(x) f_{n+q}(x) \\
f_{n+q}(x) f_{n+q+r}(x) & f_{n+p}(x) f_{n+q}(x) & \left(f_{n+q+r}(x)+f_{n+p}(x)\right)^{2}
\end{array}\right| \\
& =2 f_{n+p}(x) f_{n+q}(x) f_{n+q+r}(x)\left(f_{n+p}(x)+f_{n+q}(x)+f_{n+q+r}(x)\right)^{3}
\end{aligned}
$$

It can be proved same as Theorem 1 .

Theorem 5: If $\mathrm{n}$ and $\mathrm{p}$ are non-negative integers, $\mathrm{q}$ is positive integer with $0 \leq \mathrm{p}<\mathrm{q}, \mathrm{r}=1$, Prove that

$$
\begin{aligned}
& \left|\begin{array}{ccc}
f_{n+p}(x) & f_{n+q}(x) & f_{n+q+r}(x) \\
f_{n+p}(x)-f_{n+q}(x) & f_{n+q}(x)-f_{n+q+r}(x) & f_{n+q+r}(x)-f_{n+p}(x) \\
f_{n+q}(x)+f_{n+q+r}(x) & f_{n+p}(x)+f_{n+q+2}(x) & f_{n+p}(x)+f_{n+q}(x)
\end{array}\right| \\
& =f_{n+p}^{3}(x)+{f_{n+q}}^{3}(x)+f_{n+q+r}{ }^{3}(x) \\
& -3 f_{n+p}(x) f_{n+q}(x) f_{n+q+r}(x)
\end{aligned}
$$

It can be proved same as Theorem 1 .

Theorem 6: If $\mathrm{n}$ and $\mathrm{p}$ are non-negative integers, $\mathrm{q}$ and $\mathrm{s}$ are positive integers with $0 \leq \mathrm{p}<\mathrm{q}, \mathrm{q}+1<\mathrm{s} r=1$, Prove that

$$
\begin{aligned}
& \left|\begin{array}{cccc}
f_{n+s}(x) & f_{n+q+r}(x) & f_{n+q}(x) & f_{n+p}(x) \\
f_{n+q+r}(x) & f_{n+s}(x) & f_{n+p}(x) & f_{n+q}(x) \\
f_{n+q}(x) & f_{n+p}(x) & f_{n+s}(x) & f_{n+q+r}(x) \\
f_{n+p}(x) & f_{n+q}(x) & f_{n+q+r}(x) & f_{n+s}(x)
\end{array}\right| \\
& =x^{2}\left[f_{n+p}(x)+f_{n+q}(x)+f_{n+q+r}(x)+f_{n+s}(x)\right](2.21) \\
& {\left[f_{n+q+r}^{2}(x)-f_{n+q}^{2}(x)\right]}
\end{aligned}
$$

Proof: Assume $f_{n+p}(x)=a, f_{n+q}(x)=b$, then by (1.1) $f_{n+q+r}(x)=a+b x \quad$ and $\quad f_{n+q+r}(x)=x f_{n+q}+f_{n+p} \quad$, $f_{n+s}(x)=x f_{n+q+r}+f_{n+q}$,

It can be proved same as Theorem 1 .

Corollary 6.1: If we put $\mathrm{x}=1$ in above result, we get 


$$
\begin{aligned}
& \left|\begin{array}{cccc}
F_{n+s}(x) & F_{n+q+r}(x) & F_{n+q}(x) & F_{n+p}(x) \\
F_{n+q+r}(x) & F_{n+s}(x) & F_{n+p}(x) & F_{n+q}(x) \\
F_{n+q}(x) & F_{n+p}(x) & F_{n+s}(x) & F_{n+q+r}(x) \\
F_{n+p}(x) & F_{n+q}(x) & F_{n+q+r}(x) & F_{n+s}(x)
\end{array}\right|(2.22) \\
& =\left[F_{n+p}(x)+F_{n+q}(x)+F_{n+q+r}(x)+F_{n+s}(x)\right] \\
& F_{n+p}(x)\left[4 F_{n+q}^{2}(x)-F_{n+p}^{2}(x)\right] \\
& =F_{2(n+p)+6}(x) \cdot F_{2(n+p)}(x) .
\end{aligned}
$$

It can be proved easily.

Theorem 7: If $\mathrm{n}$ and $\mathrm{p}$ are non-negative integers, $\mathrm{q}$ is positive integer with $0 \leq \mathrm{p}<\mathrm{q}, \mathrm{r}=1$ and $\alpha=f_{n+p}(x)$, $\beta=f_{n+q}(x), \gamma=f_{n+q+r}(x)$, Prove that

$$
\begin{aligned}
& \left|\begin{array}{ccccc}
\alpha \gamma+\beta^{2} & \alpha^{2} & \beta^{2} & \alpha \gamma^{2} & -\left(\alpha \gamma+\beta^{2}\right) \\
\alpha \gamma+\beta^{2} & \alpha \gamma & -\alpha \gamma & \alpha \gamma^{2} & \alpha \gamma+\beta^{2} \\
0 & 2 \alpha \gamma & 2 \alpha \gamma & -2 \alpha \gamma^{2} & 0 \\
\alpha \gamma+\beta^{2} & -\alpha \gamma & \alpha \gamma & -\alpha \gamma^{2} & \alpha \gamma+\beta^{2} \\
-\left(\alpha \gamma+\beta^{2}\right) & \alpha^{2} & \beta^{2} & \alpha \gamma^{2} & \alpha \gamma+\beta^{2}
\end{array}\right| \\
& =-32 \alpha^{2} \gamma^{3}\left(\alpha \gamma+\beta^{2}\right) \\
& =-32 f_{n+p}{ }^{2}(x) f_{n+q+r}{ }^{3}(x) \\
& {\left[f_{n+p}(x) f_{n+q+r}(x)+f_{n+q}{ }^{2}(x)\right]^{3} .}
\end{aligned}
$$

Proof: Assume $f_{n+p}(x)=a, f_{n+q}(x)=b$, then by (1.1) $f_{n+q+r}(x)=a+b x$.

It can be proved same as Theorem 1 .

\section{Conclusion}

This paper describes determinant identities of Fibonacci polynomials. Determinants identities included various pattern of Fibonacci polynomials. Few results connected with Lucas polynomials. More identities can be developed with generalized polynomials and other classical polynomials.

\section{References}

[1] A. Lupas, "A Guide of Fibonacci and Lucas Polynomials," Octagon Math. Mag., 7 (1), 2-12, 1999.

[2] A. Benjamin, N. Cameron and J. Quinn, "Fibonacci DeterminantsA Combinatorial Approach," Fibonacci Quarterly, 45 (1), 39-55, 2007.

[3] B. Singh, O. Sikhwal and S. Bhatnagar, "Fibonacci-Like Sequence," International Journal of Advanced Mathematical Sciences, 1 (3), 145-151, 2013.

[4] B. Singh, O. Sikhwal and S. Bhatnagar, "Generalized Fibonacci Sequence and its Properties," Open Journal of Mathematical Modeling, 1 (6), 194-202, 2013.

[5] B. Singh, O. Sikhwal and Y. K. Panwar, "Generalized Determinantal Identities Involving Lucas Polynomials," Applied Mathematical Sciences, 3 (8), 377-388, 2009.

[6] Beverage David, "A Polynomial Representation of Fibonacci Numbers," Fibonacci Quarterly, 9, 541-544, 1971.

[7] Krattenthaler, "Advanced determinant calculus," Seminaire Lotharingien Combin, Article, b42q, 67, 1999.

[8] C. Krattenthaler, "Advanced determinant calculus: A Complement," Liner Algebra Appl., 411, 68-166, 2005.

[9] E. Weisstein et al., "Fibonacci number from MathWorld- A Wolfram Web Resource," http://mathworld.wolfram.com/FibonacciNumber.html

[10] J.M. Patel, "Problem H-635," Fibonacci Quarterly, 44 (1), 91, 2006.

[11] M. Bicknell-Johnson and C. Spears, "Classes of Identities for the Generalized Fibonacci number $\mathrm{G}_{\mathrm{n}}=\mathrm{G}_{\mathrm{n}-1}+\mathrm{G}_{\mathrm{n}-2}$ from Matrices with Constant valued Determinants," Fibonacci Quarterly, 34, 121-128, 1996.

[12] N. Cahill and D. Narayan, "Fibonacci and Lucas numbers Tridigonal Matrix Determinants," Fibonacci Quarterly, 42, 216221, 2004.

[13] O. Sikhwal, Generalization of Fibonacci Sequence: An Intriguing Sequence, Lap Lambert Academic Publishing GmbH \& Co. KG, Germany, 2012.

[14] S. Basir and V. Hoggatt, Jr., "A Primer on the Fibonacci Sequence Part II," Fibonacci Quarterly, 1, 61-68, 1963.

[15] S. L. Basin, "The appearance of Fibonacci Numbers and the Q Matrix in Electrical Network Theory," Mathematics Magazine, 36 (2), 84-97, 1963.

[16] T. Koshy, Fibonacci and Lucas Numbers With Applications, John Wiley and Sons, New York, 2001.

[17] V.N. Mishra, H.H. Khan, K. Khatri and L. N. Mishra, "Hypergeometric Representation for Baskakov-Durrmeyer-Stancu Type Operators," Bulletin of Mathematical Analysis and Applications, 5 (3), 18-26, 2013. 